\title{
On the structure of the turbulent interstellar atomic hydrogen
}

\section{Physical characteristics. Influence and nature of turbulence in a thermally bistable flow}

\author{
P. Hennebelle ${ }^{1}$ and E. Audit ${ }^{2}$ \\ ${ }^{1}$ Laboratoire de radioastronomie millimétrique, UMR 8112 du CNRS, École normale supérieure et Observatoire de Paris, \\ 24 rue Lhomond, 75231 Paris Cedex 05, France \\ e-mail: patrick.hennebelle@ens.fr \\ 2 Service d'Astrophysique, CEA/DSM/DAPNIA/SAp, C. E. Saclay, 91191 Gif-sur-Yvette Cedex, France \\ e-mail: edouard.audit@cea.fr
}

Received 28 July 2006 / Accepted 13 December 2006

\section{ABSTRACT}

\begin{abstract}
Aims. We study in some details the statistical properties of the turbulent 2-phase interstellar atomic gas. Methods. We present high resolution bidimensional numerical simulations of the interstellar atomic hydrogen which describe it over 3 to 4 orders of magnitude in spatial scales.

Results. The simulations produce naturally small scale structures having either large or small column density. It is tempting to propose that the former are connected to the tiny small scale structures observed in the ISM. We compute the mass spectrum of CNM structures and find that $\mathcal{N}(M) \mathrm{d} M \propto M^{-1.7} \mathrm{~d} M$, which is remarkably similar to the mass spectrum inferred for the CO clumps. We propose a theoretical explanation based on a formalism inspired from the Press \& Schecter $(1974$, ApJ, 187, 425) approach and use the fact that the turbulence within WNM is subsonic. This theory predicts $\mathcal{N}(M) \propto M^{-5 / 3}$ in $2 \mathrm{D}$ and $\mathcal{N}(M) \propto M^{-16 / 9}$ in $3 \mathrm{D}$. We compute the velocity and the density power-spectra and conclude that, although the latter is rather flat, as observed in supersonic isothermal simulations, the former follows the Kolmogorov prediction and is dominated by its solenoidal component. This is due to the bistable nature of the flow which produces large density fluctuations, even when the rms Mach number (of WNM) is not large. We also find that, whereas the energy at large scales is mainly in the WNM, at smaller scales, it is dominated by the kinetic energy of the CNM fragments.

Conclusions. We find that turbulence in a thermally bistable flow like the atomic interstellar hydrogen, is somehow different from turbulence in a supersonic isothermal gas. In a companion paper, we compare the numerical results with atomic hydrogen observations and show that the simulations well reproduce various observational features.
\end{abstract}

Key words. hydrodynamics - instabilities - ISM: kinematics and dynamics - ISM: structure - ISM: clouds

\section{Introduction}

The neutral atomic gas $(\mathrm{HI})$ is ubiquitous in the galaxies and is of great importance for the star formation process, since molecular clouds form by contraction of HI. As such, HI has been extensively observed over the years (e.g. Kulkarni \& Heiles 1987; Dickey \& Lockmann 1990; Heiles \& Troland 2003, 2005; Miville-Deschênes et al. 2003). Although HI has also received a lot of attention from the theoretical point of view (Field 1965; Field et al. 1969; Zel'dovich \& Pikel'ner 1969; Penston \& Brown 1970; Wolfire et al. 1995, 2003) and in spite of the early recognition that $\mathrm{HI}$ is a turbulent medium (e.g. Crovisier 1981; Heiles \& Troland 2005), it is only recently that the dynamical properties of HI have been investigated.

Two lines have been simultaneously pursued by various teams. Attempting to understand the properties of HI on large scales, Gazol et al. (2001, 2005), Dib \& Burkert (2005), de Avillez \& Breitschwerdt (2005), have performed 2D or 3D numerical simulations of turbulent multi-phase flows subject to various forcing. On smaller scales, various studies attempting to resolve the physical scales involved in the problem, have focused on the description of the thermal contraction, trying to understand the cooling and the condensation of a piece of WNM into CNM accurately. Hennebelle \& Pérault (1999, 2000) have studied the influence of a converging flow, Koyama \& Inutsuka (2000) have investigated the influence of a shock propagating in a bistable flow whereas Sánchez-Salcedo et al. (2002) have considered initially unstable gas. More recently, Hennebelle \& Passot (2006) have investigated the influence of Alfvén waves propagating into the medium. Because of the high numerical resolution required to treat the problem, these works were performed in only one dimension. Further important extensions of these works have been performed in 2 or 3D. Koyama \& Inutsuka (2002) have generalized their study in 2D and Kritsuk $\&$ Norman (2002) have computed the evolution of thermally unstable gas using 3D simulations.

In a recent paper (Audit \& Hennebelle 2005, here after Paper I), we have investigated the dynamical evolution of colliding flows of WNM, paying particular attention to the effect of turbulence. The main conclusions of this study are as follows: first, we confirm, in a context where the flow is strongly turbulent, the result obtained by Koyama \& Inutsuka (2002) in their study of shock propagation in WNM. The CNM is very fragmented in small clouds which have a dispersion velocity equal to a fraction of the WNM sound speed. Second, as in Gazol et al. (2001), although at much smaller scales, we find large fractions 
of thermally unstable gas and we show that turbulence is able to stabilize transiently the thermally unstable gas. Third, we show that in spite of the strong turbulence, the CNM fragments are generally not very far from pressure equilibrium with the surrounding WNM. Colliding flows of atomic interstellar gas have been further investigated by Heitsch et al. $(2005,2006)$ and by Vázquez-Semadeni et al. (2006).

One of the crucial issues in modeling atomic hydrogen is the numerical resolution which is very demanding (see Sect. 2.3). In this paper, we present the result of very high resolution numerical simulations allowing description of scales ten times smaller than in Paper I. We perform a set of simulations with various numerical resolutions to study its influence. We also study the influence of thermal conduction by varying its value. The high resolution allows a better description of the flow down to much smaller scales and offers the possibility to perform various statistical studies of the flow and CNM structures properties.

In a companion paper (Hennebelle et al. 2007, Paper III), we make preliminary comparisons between the 2D simulations presented here and various observational results. In particular, the high resolution permits the comparison with various small scale structures that have been observed in HI, like the so-called tiny small atomic structure (TSAS) and recent low column density clouds observed by Braun \& Kanekar (2005) and Stanimirović $\&$ Heiles (2005).

In Sect. 2, we describe the numerical setup and method and discuss briefly the various scales which have to be adequately treated. In Sect. 3, we present one typical timestep of the simulation and discuss qualitatively various features of the flow which are worth quantifying. Section 4 presents the density and the pressure PDF. Section 5 presents density and velocity powerspectra as well as the energy spectrum. In Sect. 6, the mass spectrum of the CNM structures in the simulation is computed and in Sect. 7, a theoretical explanation is proposed. Section 8 concludes the paper.

\section{Initial conditions and method}

The initial conditions and the methods are very similar to those of Paper I. Here, we describe them briefly for self-consistency.

\subsection{Equations, notations and numerical method}

We consider the usual fluid equations for a radiatively cooling gas including thermal conductivity namely,

$\partial_{t} \rho+\nabla \cdot[\rho u]=0$,

$\partial_{t} \rho u+\nabla \cdot[\rho u \otimes u+P]=0$,

$\partial_{t} E+\nabla \cdot[u(E+P)]=-\mathcal{L}(\rho, T)+\nabla(\kappa(T) \nabla T)$.

$\rho$ is the mass density, $u$ the velocity, $P$ the pressure, $E$ the total energy and $\mathcal{L}$ the cooling function which includes Lyman- $\alpha$, $C^{+}$and $O$ line cooling and grains photoelectric heating. The gas is assumed to be a perfect gas with $\gamma=5 / 3$ and with a mean molecular weight $\mu=1.4 m_{\mathrm{H}}$, where $m_{\mathrm{H}}$ is the mass of the proton. $\kappa$ is the thermal conductivity and is given by $\kappa(T)=\gamma C_{v} \eta(T)$ where $C_{v}=k_{\mathrm{b}} / m_{\mathrm{H}} /(\gamma-1), \eta=5.7 \times 10^{-5}(\mathrm{~T} / 1 \mathrm{~K})^{1 / 2} \mathrm{~g} \mathrm{~cm}^{-1} \mathrm{~s}^{-1}$ and $k_{\mathrm{b}}$ is the Boltzmann constant.

We use the HERACLES code to perform the simulation. This is a second order Godunov type hydrodynamical code. Godunov methods are now widely used and tested and are particularly well suited to the treatment of shocks (e.g. Toro 1997). The size of the computational domain is $20 \mathrm{pc}$ and the resolution ranges from $600^{2}$ to $10000^{2}$ cells leading to a spatial resolution ranging from $3.3 \times 10^{-2} \mathrm{pc}$ to $2 \times 10^{-3} \mathrm{pc}$.

\subsection{Initial and boundary conditions}

The boundary conditions consist in an imposed converging flow at the left and right faces of amplitude $1.5 \times C_{s, \mathrm{wnm}}$ on top of which turbulent fluctuations of amplitude $\epsilon=2$ (see Paper I for accurate definitions) have been superimposed. On top and bottom faces, outflow conditions have been setup. This means that the flow is free to escape the computational box across these 2 faces.

The initial conditions consist of uniform WNM $(n \simeq$ $0.8 \mathrm{~cm}^{-3}$ ) at thermal equilibrium. It is worth stressing that initially no CNM is present in the computational box. The simulations are then runned until a statistically stationary state is reached. Typically, this requires about 5 to 10 box crossing times. At this stage, the incoming flow compensates on average the outgoing material.

For the highest resolution runs, this would lead to very long simulations and we therefore adopt a slightly different strategy. We start the run with a lower resolution of $1250^{2}$ cells and wait until the statistically stationary regime is obtained. Then, we increase the resolution of the simulation by a factor 2 and restart the now 4 times larger simulation. This new simulation is runned until the stationary regime is reached. Typically this takes about two box crossing time. This procedure is then repeated until the desired spatial resolution is obtained.

\subsection{Scales}

Contrary to polytropic fluids, a 2-phase medium has various spatial and temporal characteristic scales which have to be adequately described. Here, we briefly recall them.

\subsubsection{A static spatial scale}

The Field length (Field 1965) is the length at which thermal diffusivity becomes comparable to the heating and cooling terms. Its typical value is about $0.1 \mathrm{pc}$ in the WNM and about $10^{-3} \mathrm{pc}$ in the CNM. The Field length is also the typical scale of the thermal fronts that connects the cold and warm phases. As demonstrated by Field (1965), it determines the smallest wavelength at which thermal instability can grow and, therefore, the size of the smallest CNM structures. The effect of varying the thermal diffusivity is discussed in the following.

\subsubsection{Three dynamical scales}

We distinguish three scales whose origin is due to dynamical processes. The first scale is the cooling length of the WNM, i.e. the product of the cooling time and the sound speed within WNM, $\lambda_{\text {cool }}=\tau_{\text {cool }} \times C_{\text {s,WNM. This scale is about } 10 \mathrm{pc} \text { and }}$ corresponds to the typical length at which WNM is non linearly unstable and can be dynamically triggered by a compression into the unstable regime.

Since the ratio between the CNM and WNM density is about hundred, the size of a CNM structure formed through the contraction of a piece of WNM of size $\lambda_{\text {cool }}$, will be typically, hundred time smaller (assuming a monodimensional compression). Therefore, the size of the CNM structures is about the 
cooling length of WNM divided by hundred, leading to about $\lambda_{\text {cool }} / 100 \simeq 0.1 \mathrm{pc}^{1}$.

Finally, as first pointed out by Koyama \& Inutsuka (2002), the fragments of CNM have a velocity dispersion equal to a fraction of the sound speed of the medium in which they are embedded, i.e. WNM. Since the contrast between the sound speed within the two phases is about 10, it means that CNM structures undergo collisions at Mach number, $\mathcal{M} \simeq 10$. If, for simplicity, we assume isothermality and apply Rankine-Hugoniot relations, we obtain that the size of the shocked CNM structures is given by the size of the CNM structures divided by $\mathcal{M}^{2}$ which is about $10^{-3} \mathrm{pc}$, whereas the density peak is given by $100 \times$ $\mathcal{M}^{2} \simeq 10^{4} \mathrm{~cm}^{-3}$. Note that since the effective polytropic index of the CNM, $\gamma_{\mathrm{eff}}$, is closer to 0.7 than to 1 , these numbers are underestimated by a factor of about 7 .

The consequences of not solving properly these scales have been previously discussed qualitatively in Paper I. In the following, quantitative estimates are given.

\subsection{Comparison with other simulations}

As explained in the previous section, it is actually not possible to treat simultaneously all the scales involved in the physics of the atomic gas. This implies compromises and induces various choices of initial, boundary and forcing conditions. In order to make clear the domain of validity of various related works, we now discuss the numerical and physical setups which have been chosen. It is worth stressing that the different choices are complementary and equally interesting since they allow one to focus on different aspects of the same physical problem.

Koyama \& Inutsuka (2002) have investigated the propagation of a shock into WNM. They have an extremely high spatial resolution $\left(7 \times 10^{-4} \mathrm{pc}\right)$ and a relatively modest box size of about $0.25 \mathrm{pc}$ (in the transverse direction). With these choices, the CNM structures are very well resolved but the statistics are low and the structures are small. In a recent paper (Koyama \& Inutsuka 2006), they consider a larger box and try to understand the properties of the turbulence that develops without any external forcing.

Heitsch et al. (2005, 2006) and Vázquez-Semadeni et al. (2006) have focused on the formation of a CNM turbulent layer. They consider a simulation box of about $\simeq 10 \mathrm{pc}$ and have a resolution of about $10^{-2} \mathrm{pc}$. Such a setup which is very similar to the one used in Paper I, allows to treat reasonably well the large scales, but does not provide a description of the CNM structures as accurate as the CNM structures obtained in Koyama \& Inutsuka (2002). Also in these work, the authors focus on the transient regime, rather than on a time-independent situation.

Gazol et al. (2001, 2005) and Piontek \& Ostriker (2004) have focused on larger simulation boxes $(100 \mathrm{pc}-1 \mathrm{kpc})$ and coarser spatial resolution $(0.5-5 \mathrm{pc})$. The same kind of scales has been investigated by de Avillez \& Breitschwerdt (2005) using an AMR scheme. This leads to a much better description of the larger scales and a more realistic self-consistent forcing (e.g. due to supernovae or the development of the magneto-rotational instability) that the choice makes in this work, but only marginal description of CNM structures can be achieved.

${ }^{1}$ In fact, as we will see in the following, the size of the CNM structures follows a density distribution. The numbers given here are simply indicative

\section{Results}

In this section, we present results for the $10000^{2}$ cells simulation which corresponds to the highest numerical resolution runs. We discuss qualitatively the main properties of the 2-phase turbulent flow and introduce the most important aspects which are studied quantitatively in the following and in Paper III.

\subsection{Main features}

Figure 1 displays the density and velocity fields of the simulation at one representative timestep (i.e. well after statistically stationary state has been reached) whereas Fig. 2 shows a spatial zoom of $5 \mathrm{pc}$.

With our choice of boundary conditions which entails a converging flow, the formation of a layer-like structure is triggered in the middle of the computational box ( $x \simeq 5 \mathrm{pc}, y \simeq 2-18 \mathrm{pc}$ ).

The fragmentation of such a layer due to the development of various instabilities, like the Vichniac instability (Vichniac 1994) or the Kelvin-Helmholtz instability has been studied by Walder \& Folini $(1996,1998)$ and Heitsch et al. $(2005,2006)$ for a radiatively cooling gas and by Folini \& Walder (2006) for strongly supersonic isothermal gas. Here, since the converging flow imposed at the boundary presents strong velocity fluctuations, the turbulence and the fragmentation of the layer are not easily attributable to a single instability and at least, partly due to the driven turbulence. Indeed, in Paper I, we vary the amplitude of the shear velocity fluctuations which are superimposed to the converging flow. As expected, we find that the stronger these fluctuations, the stronger the turbulence in the computational domain. Near the boundaries of the computational box, the structure of the flow is somehow different because the converging flow is concentrated in the middle of the computational box faces, whereas the ram pressure at the edge of the box faces almost vanishes.

The overall structure of the flow appears to be highly complex. The cold phase is very fragmented and the two phases are highly intervowen. There are large regions of space where there is only WNM and regions where most of the mass is obviously in the CNM. However, even in these denser regions, WNM is still present and intrusive. Only few large CNM structures have formed, most of them appear to be very small. This is more clearly seen in Fig. 2 which also reveals that the structures are often connected to each other by filaments of lower density and that the density of the CNM varies over 2 orders of magnitudes.

\subsection{Shocked CNM: TSAS?}

While most of the CNM structures have a density of about $100 \mathrm{~cm}^{-3}$ (e.g. structure located at $(x, y)=(3,8) \mathrm{pc}$ ), shocked regions present densities up to $\simeq 10^{4} \mathrm{~cm}^{-3}$ (e.g. structure located at $(x, y)=(1.66,7.07) \mathrm{pc})$. This is more clearly seen in Fig. 4 which shows a spatial zoom of Fig. 2 and in Fig. 5 where a cut along the $y$-axis is displayed. These two figures also reveal that the density peak corresponds to a peak of pressure reaching a value of about $10^{5} \mathrm{~K} \mathrm{~cm}^{-3}$ and that this strong fluctuation has been induced by a converging flow of velocity $\pm 5 \mathrm{~km} \mathrm{~s}^{-1}$. Altogether, this is perfectly consistent with the orders of magnitude given in Paper I and recalled in Sect. 2.3 to characterize the shocked CNM structures. The density $\left(\simeq 10^{4} \mathrm{~cm}^{-3}\right)$ and the size $(\simeq 400 \mathrm{AU})$ appear to be close to what is inferred for the TSAS structures observed in the CNM (e.g. Heiles 1997), although the more extreme events present densities up to 10 times this value and scales roughly 10 times smaller. It is therefore tempting to propose that TSAS 


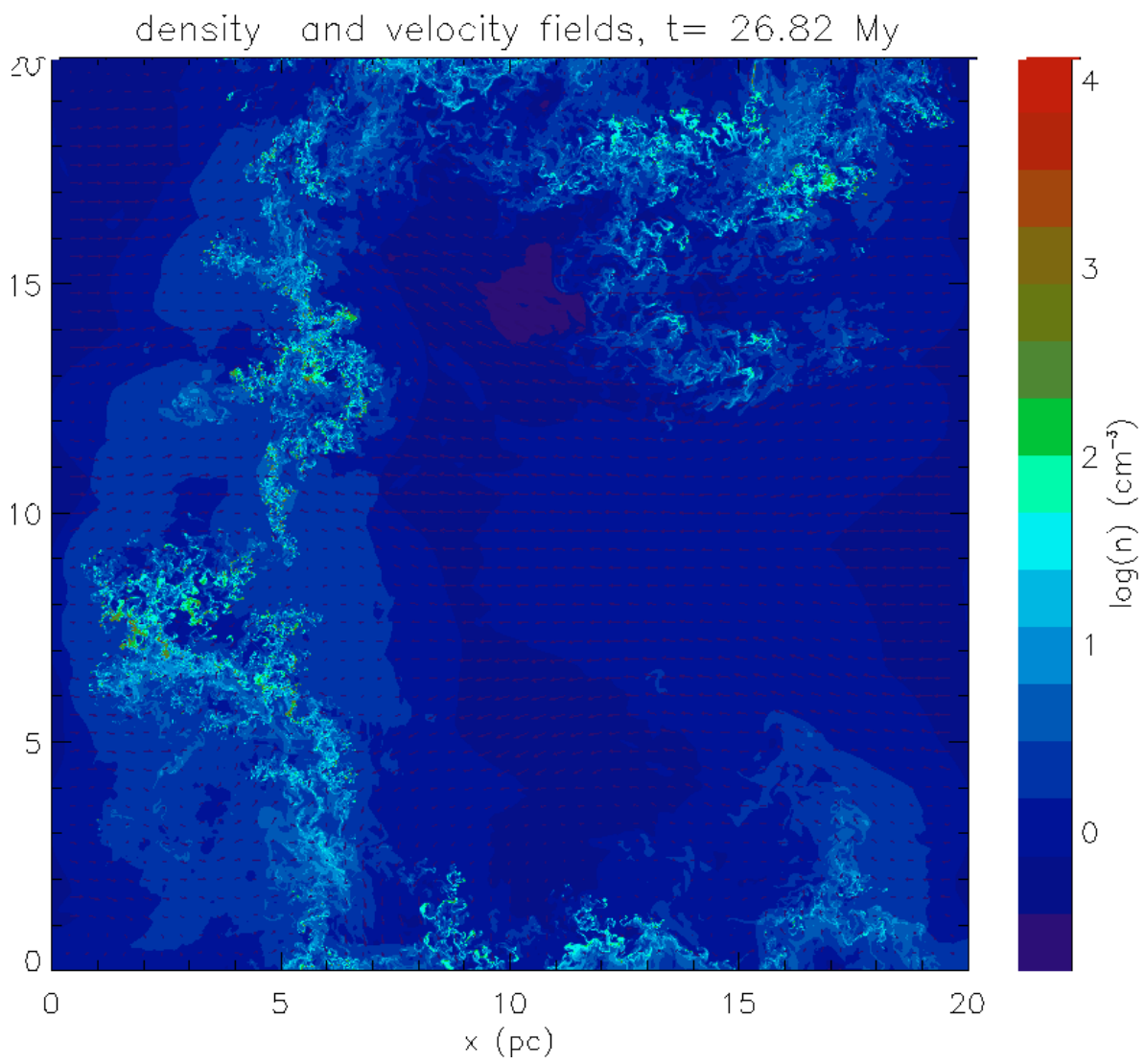

Fig. 1. Density and velocity fields at one time step of the $10000^{2}$ cells simulation corresponding to a spatial resolution of $2 \times 10^{-3} \mathrm{pc}$. Note that because of the large dynamics, the arrows are not easily seen in the CNM clouds. are produced by supersonic collisions between CNM fragments. It should be noted that the density peak is not fully resolved because of insufficient numerical resolution and therefore it is expected that this event should have indeed a larger peak density and a smaller size if the numerical resolution was higher. It is also important to emphasize that the cooling function used in this simulation is probably not accurate for such high densities.

\subsection{Small column density clouds}

In a similar way, the properties of the smallest structures that form in the simulations appear to be reminiscent of the tiny structures which have been recently observed by Braun \& Kanekar (2005) and Stanimirović \& Heiles (2005). Many of them can be seen in Fig. 2 (e.g. for $y \leq 6 \mathrm{pc}$ ) and a cut through two small CNM structures is displayed in Fig. 5 ( $y \simeq 6.68$ and $6.83 \mathrm{pc}$ ). The density of these two structures is about 30 and $100 \mathrm{~cm}^{-3}$ whereas their size is about $10^{-2} \mathrm{pc}$.

Further examples are shown in Paper III in which synthetic HI spectra are also discussed.

\subsection{Pressure distribution}

Another important aspect is revealed by the pressure field displayed in Fig. 3. The pressure field appears well organized with large scale systematic gradients. At large scales, the pressure and the density fields appear to be broadly anti-correlated. Indeed, the central cloud (located roughly between $x=1$ and $x=4$ and $y=6$ and $y=9$ ) is surrounded by high pressure WNM, whereas its internal pressure is a few times lower. This is a consequence of the radiative cooling. When the WNM fluid particles enter into the shocked layer, their internal pressure goes up like in an adiabatic shock, then they cool and finally condense out. Indeed, the cooling length of shocked (Mach 1.5-2) WNM is few parsec which is the length of the high pressure WNM layer seen on the left part of the box. Interestingly, the high density shocked CNM is located at the interface between the high pressure WNM and the low pressure cloud. Note that since the WNM pressure $\left(P \simeq 10^{4} \mathrm{~K} \mathrm{~cm}^{-3}\right)$ is lower by at least one order of magnitude than the large pressure fluctuation $\left(P \simeq 10^{5} \mathrm{~K} \mathrm{~cm}^{-3}\right)$, it cannot be the triggering agent. However, this high pressure WNM is in part responsible of these large pressure fluctuations since it actively induces the formation of relatively high velocity CNM structures, which then undergo supersonic collisions.

At small scales, the situation is much different. The pressure is poorly correlated with the density field displayed in Fig. 2. Most of the CNM structures do not have high thermal pressure (see Fig. 5) with the notable exception of the densest regions which correspond to shocked CNM. Indeed, some of the CNM structures have a thermal pressure lower than the surrounding WNM as for example, the 2 structures located at $y=7.15$ and $7.3 \mathrm{pc}$ in Fig. 5. This low pressure indicates that the structures have just formed, so that there was not enough time for the initially low thermal pressure induced by the radiative cooling to readjust to the surrounding higher pressure.

Finally, the density field indicates that the CNM structures are well identified and connected to the surrounding WNM by very stiff fronts. Moreover, as in the static 2-phase medium (Field et al. 1969; Wolfire et al. 1995), most of the CNM structures are not far from quasi-pressure equilibrium with the surrounding medium (in the sense that pressure fluctuations are small with respect to the density contrast between the two phases). This is likely a consequence of the large 


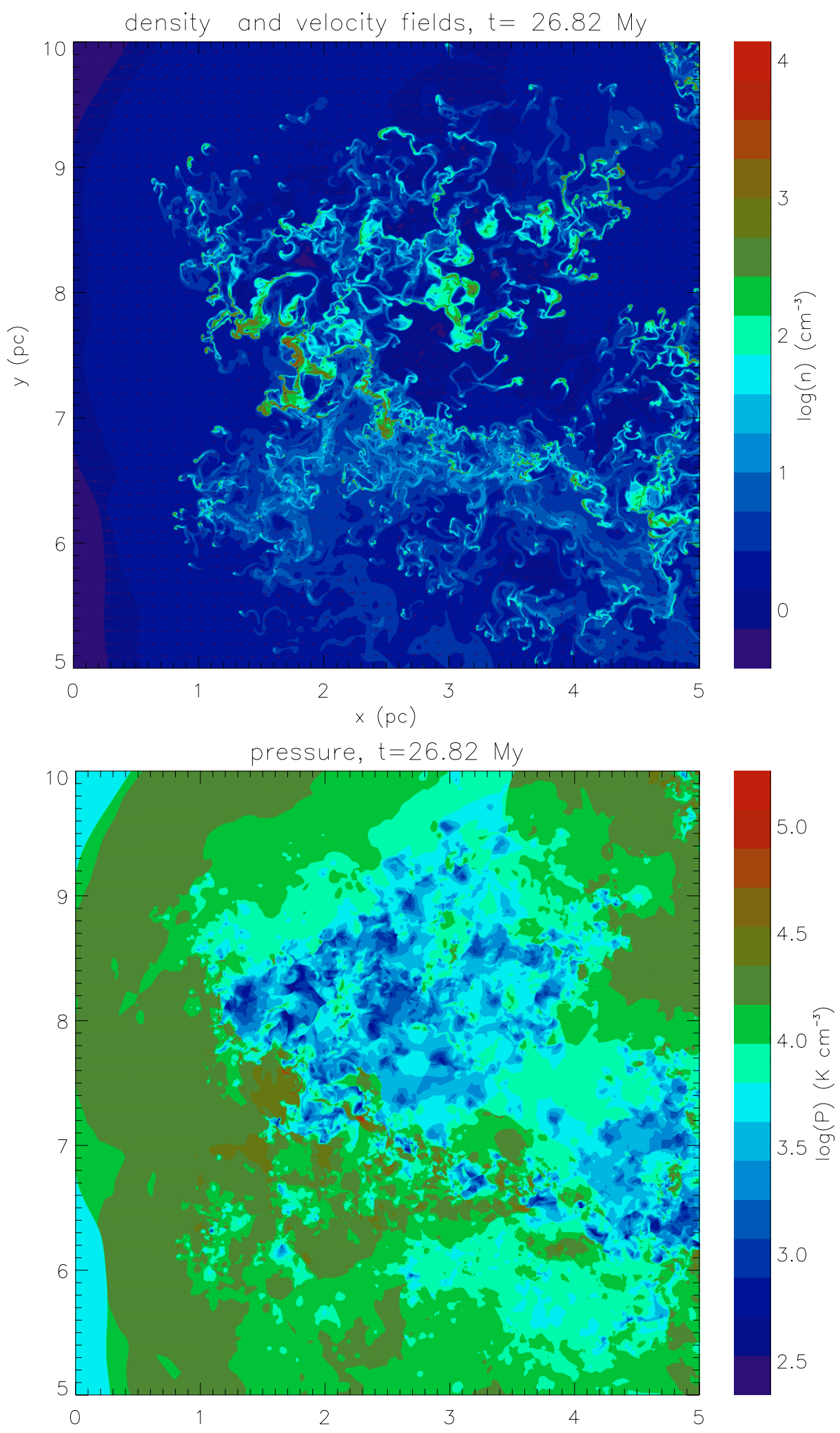

Fig. 2. Spatial zoom of the field displayed in Fig. 1.
Fig. 3. Logarithm of the pressure field (in $\mathrm{K} \mathrm{cm}^{-3}$ ) corresponding to Fig. 2. density contrast between the phases. First, with a density contrast of about hundred, the sonic waves propagating in the WNM as well as the eddies get mostly reflected when they reach a WNM/CNM interface, probably making the energy injection inside the structures relatively inefficient. Second, since the scale of the CNM structures is small compared to the scale of the
WNM flow and since most of the turbulent energy is on the largest scales, the WNM flow is relatively uniform at the scale of the CNM structures and mostly advects them uniformly.

To summarize, it seems that a turbulent 2-phase flow cannot be accurately described as a polytropic turbulent flow, neither as a static 2-phase medium, but, instead, they present a kind of 


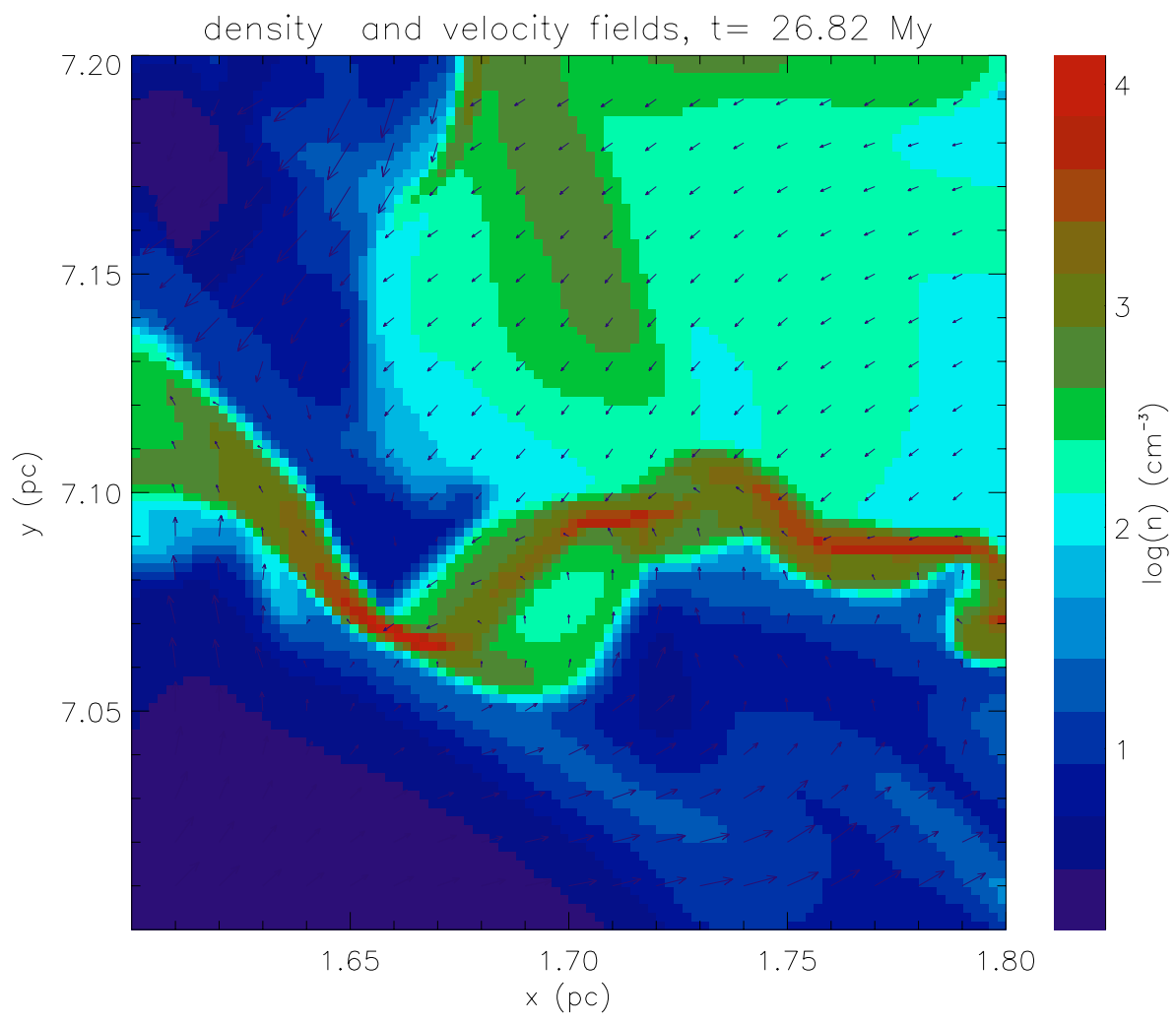

Fig. 4. Spatial zoom of the fields displayed in Fig. 1.

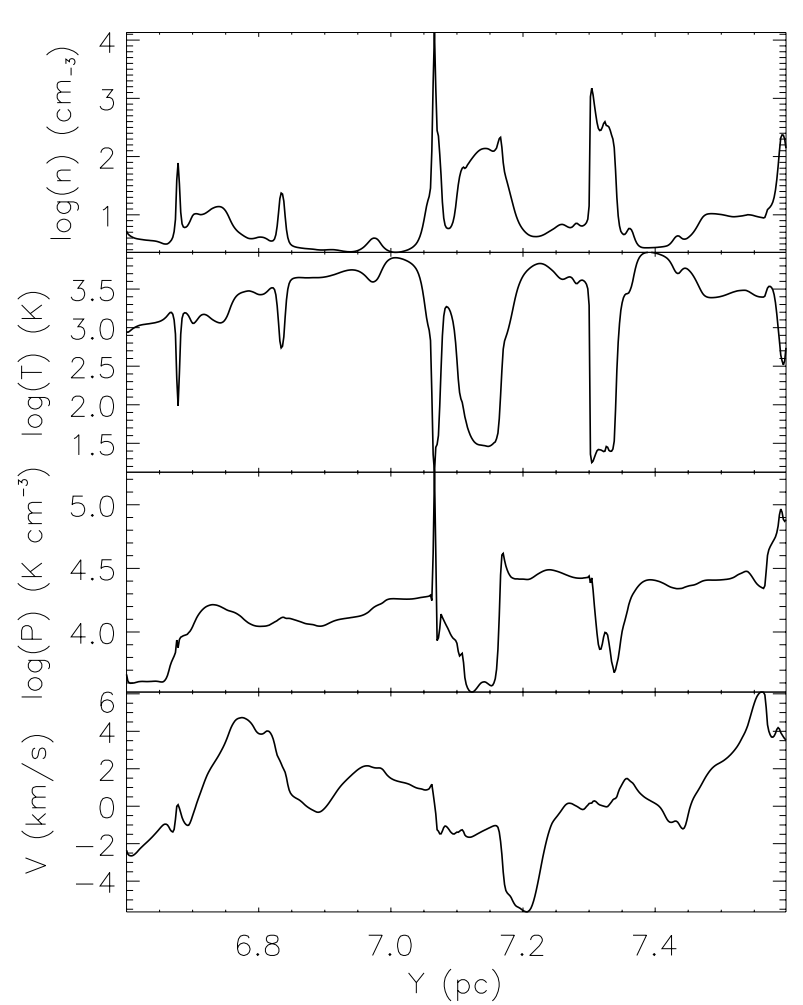

Fig. 5. Density, pressure and velocity profiles at $x=1.65 \mathrm{pc}$ for $\mathrm{y}$ ranging from 6.6 to $7.6 \mathrm{pc}$. Note that this corresponds to the line of sight which intercepts the cell having the highest density at this timestep.

duality, some aspects being more reminiscent of turbulent flows and other of static 2-phase media with fluctuations which can be up to hundred times the mean CNM density or pressure. In the following, we therefore quantify the mass distribution of

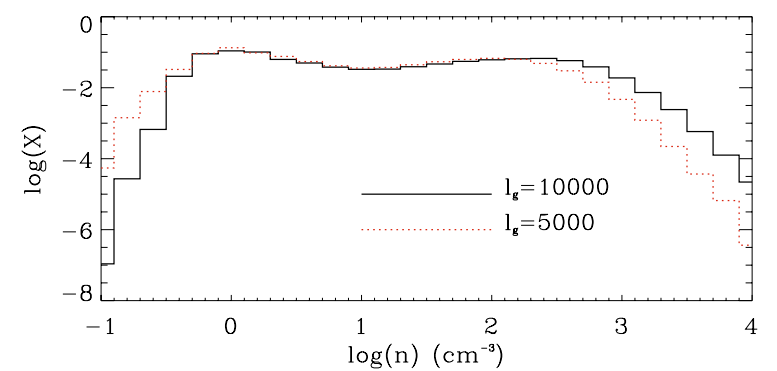

Fig. 6. Probability distribution function of the logarithm of the density field.

CNM structures, as well as the density and pressure distribution of the flow. We also study the statistics of the flow by computing various power-spectra, in order to characterize its scale dependence. In Paper III, we quantify further the numerical results by computing line of sight statistics and various properties of the CNM structures.

\section{Density and pressure probability distribution function}

Figures 6 and 7 show the probability distribution function of the logarithm of the density and pressure respectively. Full black lines correspond to the runs having $l_{g}^{2}=10000^{2}$ cells, whereas the dotted red lines are for the runs with $l_{g}^{2}=5000^{2}$ cells. The density pdf presents two peaks which correspond to the WNM and CNM densities. The pdf values at densities between 5 and 50 indicates that there is a significant fraction of thermally unstable gas, as first stated by Gazol et al. (2001) and confirmed in Paper I.

In principle, the density pdf allows estimation of the fraction of the dense gas that has been compressed by the ram pressure. 


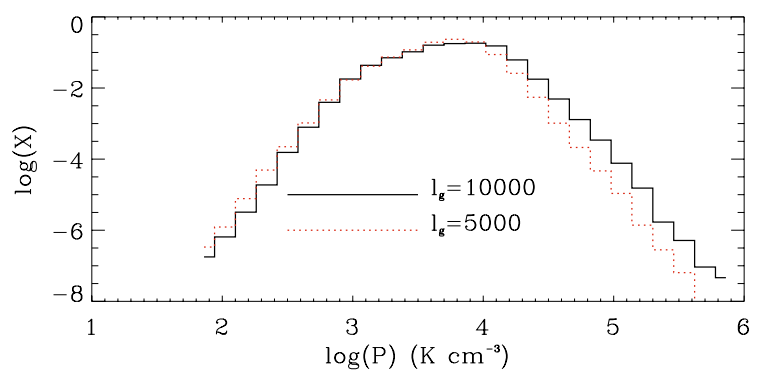

Fig. 7. Probability distribution function of the logarithm of the pressure field.

However it is seen that the results for the high density part do clearly depend on the numerical resolution, meaning that higher resolution runs will lead to a higher fraction of high density gas. It should also be clear that since the fraction of high density gas depends on the cooling processes, it will certainly be modified by a more accurate treatment of the microphysics. Having these limitations in mind, we can indicatively say that the fraction of gas denser than $\simeq 10^{3} \mathrm{~cm}^{-3}$ is about $1-3 \%$ for this simulation. It is important to keep in mind that, as emphasized in Paper I and in Gazol et al. (2005), this obviously depends on the driving of the turbulence.

The pressure pdf indicates that the average pressure is about $6000 \mathrm{~K} \mathrm{~cm}^{-3}$, which is the pressure of the WNM injected at the boundary. The large pressures are induced by the ram pressure of the incoming flow. The pressure distribution is reminiscent of pdf found by various authors in different contexts (Passot \& Vázquez-Semadeni 1998; Scalo et al. 1998). Comparison with the results presented by Gazol et al. (2005) is more straightforward since they consider a thermally bistable flow as well. As in their case, we find that a near-powerlaw tail develops at high and low pressure (in their case they report that this depends on the driving length). Because of the higher numerical resolution, we reach higher values of pressure. Our pressure pdf appears to be somehow similar to the results presented in their Fig. 11 corresponding to a large scale driving. However, the significant difference between the 2 numerical experiments precludes detailed comparisons.

\section{Velocity and density power-spectra and energy spectrum}

\subsection{Incompressible and isothermal turbulence}

An important diagnostic commonly used in fluid dynamics to characterize the scale-dependent structure of the flow is the power-spectrum of the fluid variables such as the velocity and the density fields. The power-spectrum equal to the square of the module of the Fourier transform, depends on all components of the wave vector $\boldsymbol{k}$. The shell average power-spectrum is the averaged of the power-spectrum in spherical shells in $k$-space. In the following, the power-spectrum will refer to shell averaged power-spectrum.

In incompressible turbulence, the velocity power-spectrum, $P_{V}$, is related to the energy spectrum as $E(k) \propto k^{D-1} P_{V}$, where $D$ is the space dimension. The Kolmogorov (Kolmogorov 1941) prediction for the energy spectrum of homogeneous, 3D and incompressible turbulence is: $E(k) \propto k^{-5 / 3}$. Note that this corresponds to power-spectrum proportional to $P_{V} \propto k^{-11 / 3}$ for 3D flows and to $P_{V} \propto k^{-8 / 3}$ for $2 \mathrm{D}$ flows. However, the behaviour of $2 \mathrm{D}$ incompressible turbulence is very different from the 3D turbulence because of enstrophy conservation

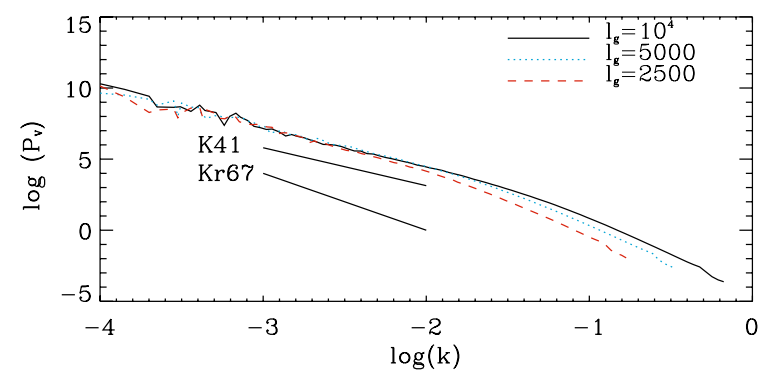

Fig. 8. Velocity power-spectrum for numerical resolutions equal to $\mathrm{d} x=$ $8 \times 10^{-3}, 4 \times 10^{-3}$ and $2 \times 10^{-3}$ pc.

(Kraichnan 1967). At scales larger than the injection scale, one expects to recover the Kolmogorov energy spectrum whereas at scales smaller than the injection scale, Kraichnan (1967) predicts $k P_{V} \propto k^{-3}$.

In 1D compressible turbulence, when shocks dominate, a Burgers-like spectrum $P_{V} \propto k^{-2}$ is expected (Elsasser \& Schamel 1976). Passot et al. (1988) have confirmed, using isothermal bidimensional numerical simulations, that the compressible part of the velocity field follows the same behaviour whereas for the solenoidal part, the prediction of Kraichnan (1967) is well verified, $k P_{V} \propto k^{-3}$. Kim \& Ryu (2005) recently explored numerically in some details the 3D case. They show that the density power-spectrum $\left(P_{\rho}\right)$ for low rms Mach number flow is close to the Kolmogorov power-spectrum, whereas it is much flatter at high rms Mach number, i.e. $k^{2} P_{\rho} \propto k^{-\alpha}$ with $\alpha \simeq 0.5$ for an rms Mach equal to 12 . They interpret this result as the consequence of the density distribution being highly concentrated in sheets and filaments. Flat density powerspectra have also been observed in MHD isothermal simulations. Padoan et al. (2004) report $\alpha=0.25$ for a model with equipartition between magnetic and kinetic energy and $\alpha=0.75$ for a super-Alfvénic model (see also Beresnyak et al. 2005).

\subsection{Velocity power-spectrum}

Figure 8 shows the velocity power-spectrum for various numerical resolutions, whereas Fig. 9 shows the compensated powerspectra (i.e. $k^{a} P_{V}$ the value of $a$ being given in the figure) of the solenoidal and compressible components of the velocity field. For reference the Kolmogorov (1941) and the Kraichnan (1967) prediction have been displayed in Fig. 8. As in Passot et al. (1988), the compressible part has a power-spectrum $k P_{V} \propto k^{-2}$. The velocity power-spectrum, as well as the power-spectrum of the solenoidal component, appear to be in good agreement with the Kolmogorov law, $k P_{V} \propto k^{-5 / 3}$ for $k$ between $\simeq-3.3$ and $\simeq-1.8$.

This may be at first surprising because, as recalled above, the prediction of Kraichnan for 2D incompressible fluid is that there is an invert cascade above the energy injection scale, leading to the velocity power-spectrum predicted by Kolmogorov and a direct enstrophy cascade at scale below the injection scale leading to $k P_{V} \propto k^{-3}$. However, this result is a consequence of the conservation of vorticity in incompressible or barotropic flow. When the flow is non barotropic the vorticity equation writes:

$\partial_{t} \omega+\nabla \times(\omega \times v)=\nabla P \times \nabla\left(\frac{1}{\rho}\right)$.

In a radiatively cooling flow, as the interstellar atomic hydrogen, the pressure and the density are not necessarily correlated as already emphasized in Sect. 3. This means that the baroclinic 


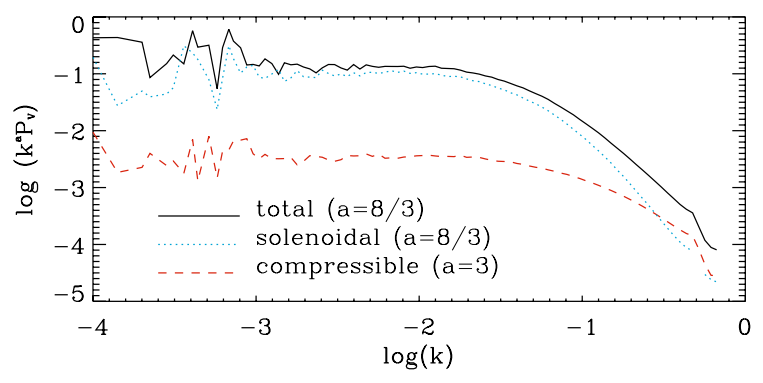

Fig. 9. Compensated power-spectrum of velocity (full line), solenoidal or shear component of velocity (dotted line), compressible component (dashed line). The spectra have been multiplied by $k^{a}$ where the value $a$ is indicated on the figure.

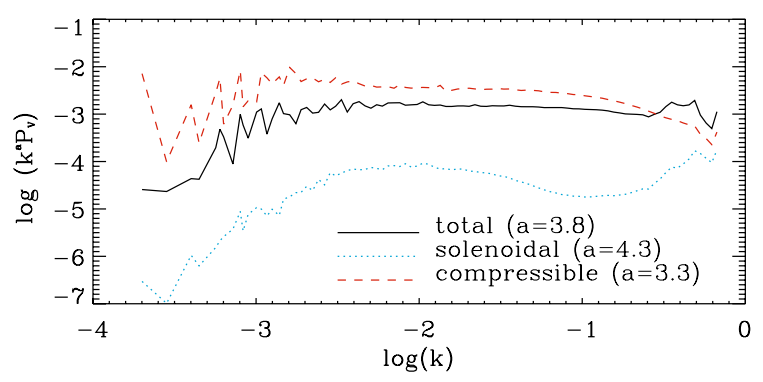

Fig. 10. Isothermal simulation. Compensated power-spectrum of velocity (full line), solenoidal or shear component of velocity (dotted line), compressible component (dashed line). The spectra have been multiplied by $k^{a}$ where the value $a$ is indicated on the figure.

term on the right hand side, is not small with respect to the second term of the left hand side, implying that enstrophy is not conserved. To verify this, we have computed the mean value of the (norm of the) baroclinic term and of the second lhs term over the whole simulation. We find that the former is on average 10 times smaller than the latter. We also find that for about $7 \%$ of our grid points, the ratio between the two is higher than 0.5 . It seems likely to us that this constitutes a sufficiently large deviation from the strict enstrophy conservation to explain the deviation from Kraichnan's prediction. Unsurprisingly, we find that the higher values of the baroclinic term are obtained in the fronts between the 2-phase where the density changes much but not the pressure.

To confirm the influence of the baroclinic term, we have performed isothermal simulations with exactly the same setup as simulations with cooling (same code and same initial and boundary conditions). In these simulations, the baroclinic term vanishes exactly. The compensated spectra are shown in Fig. 10. The power-spectrum of the solenoidal component is very stiff and roughly proportional to $k^{-4.4}$ whereas the compressible component has a power-spectrum proportional to about $k^{-3.2}$. These numbers are indeed much closer to the results of Passot et al. (1988) and to the prediction of Kraichnan (1967).

Finally, we note that, as revealed by Fig. 9, although compressible and shear modes are comparable at large scales, the second dominates at small scales for $\log (k)>-3$ (solid and dotted lines are very close between $-1>\log (k)>-3)$.

\subsection{Density power-spectrum}

Figure 11 shows the power-spectrum of the density field. It appears to be flat since one finds $k P_{\rho} \propto k^{-0.4}$. This is reminiscent of what is observed by Kim \& Ryu (2005) although in the

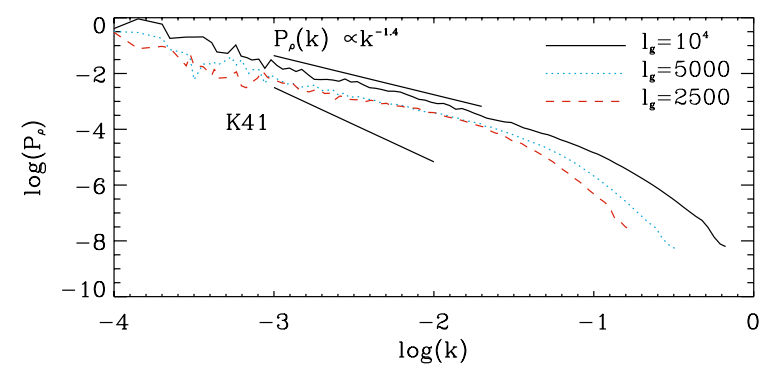

Fig. 11. Power-spectrum of density for numerical resolutions equal to $\mathrm{d} x=8 \times 10^{-3}, 4 \times 10^{-3}$ and $2 \times 10^{-3} \mathrm{pc}$.

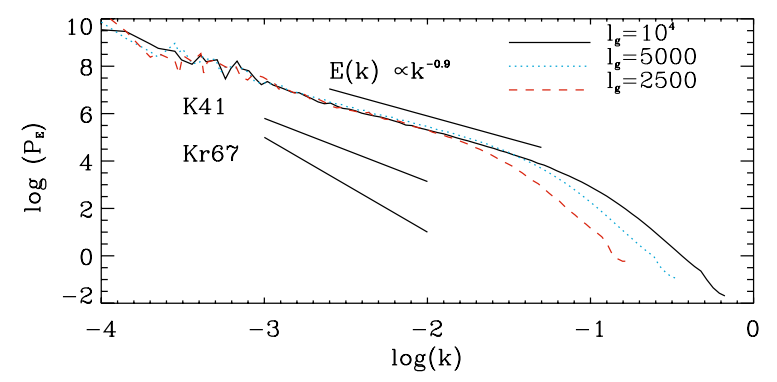

Fig. 12. Energy spectrum for numerical resolutions equal to $\mathrm{d} x=8 \times$ $10^{-3}, 4 \times 10^{-3}$ and $2 \times 10^{-3} \mathrm{pc}$.

present case the strong and stiff density fluctuations are induced by the 2-phase nature of the flow rather than by highly supersonic motions.

Kim \& Ryu (2005) proposed that the flat density powerspectrum can be understood by the fact that the Fourier transform of a Dirac function is independent of $k$ leading to $P_{\rho} \propto k^{0}$ in $1 \mathrm{D}$. We believe that this explanation is also valid in the present case and that the density power-spectrum is related to the size distribution of the structures (Figs. 15-17). Indeed since the structures are bounded by contact discontinuities, they have stiff boundaries and they resemble, depending on the scales at which one is considering them, either to the Dirac or Heaviside functions.

As a matter of fact, the end of the inertial range which occurs at about $k \simeq 0.1$ (about 10 cells in the simulation) corresponds to the length at which the structure distribution is influenced by the numerical diffusivity implying that the number of density peaks at these scales becomes gradually smaller.

Interestingly, Desphande et al. (2000) using $21 \mathrm{~cm}$ line absorption data probing linear scales between $10^{-2}$ and $3 \mathrm{pc}$, therefore comparable to the present simulation, report density power-spectrum $k^{2} P_{\rho} \propto k^{-\alpha}$, in two different regions for which $\alpha=0.75$ and $\alpha=0.5$. These values are significantly flatter than the Kolmogorov index and at least for the second one, are very close to the value of 0.4 which is inferred in our simulations.

\subsection{Energy spectrum}

Figure 12 shows the power-spectrum, $P_{E}$, of the quantity $\sqrt{\rho} v$. The quantity $k P_{E}$ corresponds to the energy spectrum in the incompressible case and following Klessen et al. (2000), we call it the energy spectrum, $E(k)$. It is rather different from the Kolmogorov prediction and significantly flatter. In the inertial range $E(k) \propto k^{-0.9}$, meaning that the energy is approximately equally distributed in the $k$-space. This appears to be a direct consequence of the density power-spectrum discussed above which relies on the strong density fluctuations due to the 2-phase 


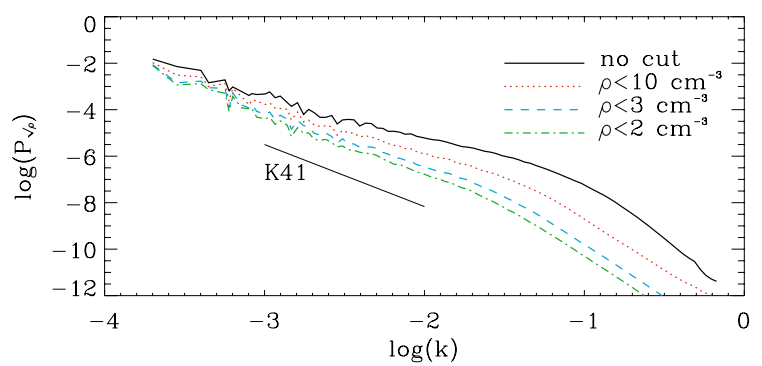

Fig. 13. Power-spectrum of the square root of density field for data obtained by taking $\min \left(\rho, \rho_{0}\right)$ for various values of $\rho_{0}$.

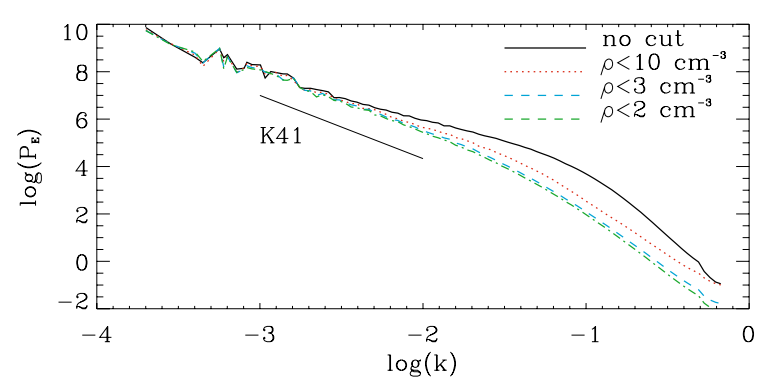

Fig. 14. Energy spectrum for data obtained by taking $\min \left(\rho, \rho_{0}\right)$ for various values of $\rho_{0}$.

nature of the flow. In order to confirm this, we have calculated the energy spectrum and the power-spectrum of the square root of $\rho$ for data obtained by taking $\min \left(\rho, \rho_{0}\right)$. The results for various values of $\rho_{0}$, namely 2,3 and $10 \mathrm{~cm}^{-3}$ as well as the result for the untruncated field, are shown in Figs. 13 and 14. When $\rho_{0}$ decreases, $P_{\sqrt{\rho}}$ becomes stiffer (note that for the untruncated field, we find $k P_{\sqrt{\rho}} \propto k^{-0.6}$ in the inertial range). The same behaviour is also observed for the energy spectrum which becomes closer to the velocity power-spectrum shown in Fig. 8 .

For $k$ larger than $\simeq 5 \times 10^{-3}$ (corresponding to a scale of about $0.5 \mathrm{pc}$ ) and $\rho_{0}<3 \mathrm{~cm}^{-3}$, the energy spectrum becomes significantly lower than the energy spectrum obtained for the untruncated field. This clearly indicates that at scales smaller than about $0.5 \mathrm{pc}$, most of the energy is stored in the CNM bulk motions. In other terms, in our numerical experiment, the 2-phase nature of the flow appears to play an important rôle and constitutes a significant deviation of single phase hydrodynamics at scales smaller or comparable to about $1 \mathrm{pc}$.

\section{Mass spectrum}

As in Paper I, we extract the structures by a simple clipping algorithm. Since the cold structures are connected to the surrounding warm gas by stiff thermal fronts such simple criteria has a clear physical meaning. The threshold is equal to $30 \mathrm{~cm}^{-3}$ which lies in the thermally unstable regime (note that we have verified that taking $10 \mathrm{~cm}^{-3}$ instead does not change the results significantly).

\subsection{Numerical result}

Figure 15 shows the number of structures, $n_{\mathrm{s}}$, per logarithmic mass interval, $\mathrm{d} n_{\mathrm{s}} / \mathrm{d} \log M$ (note that since the simulations are $2 \mathrm{D}$, the mass is expressed in solar mass per parsec). The largest structures have a mass of about one solar mass per parsec whereas the mass of the smallest structures is about $\simeq 10^{-5}$ solar mass per parsec. The number of structures, $\mathcal{N}(M)$, decreases with mass, and follows approximately: $\mathrm{d} n_{\mathrm{s}} / \mathrm{d} \log M \propto 1 / M^{\beta-1}$

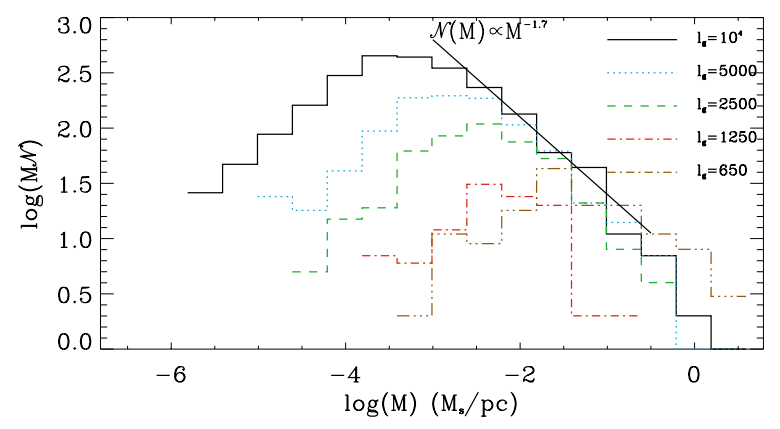

Fig. 15. Number of structures per logarithmic interval of mass (in solar mass per parsec). Various numerical resolutions are shown.

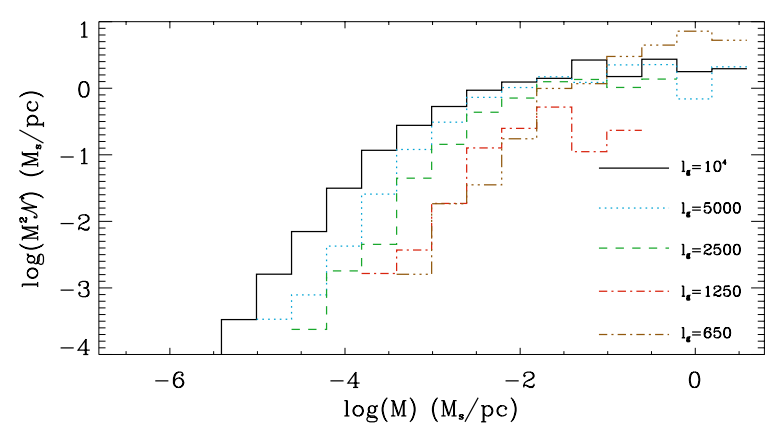

Fig. 16. Total mass within CNM structures per logarithmic interval of mass.

with $\beta \simeq 1.7$, until $\log (M) \simeq-3$. This implies that the number of structures per mass interval is $\mathrm{d} n_{\mathrm{s}} / \mathrm{d} M=\mathcal{N}(M) \propto 1 / M^{\beta}$.

For $\log (M) \leq-3, \mathrm{~d} n_{\mathrm{s}} / \mathrm{d} \log M$ increases with mass. This part of the distribution is most likely affected by the numerical resolution. This is confirmed by the fact that the numbers of low mass objects decreases with the numerical resolution. Interestingly enough, the number of massive structures becomes independent of the resolution for $\mathrm{d} x \leq 10^{-2} \mathrm{pc}$ (see solid, dotted and dashed curves). This indicates that numerical convergence is reached for the large structures $(\log (M) \gtrsim-3)$. It is however clear that no numerical convergence has yet been reached for the smallest structures. We also note that the number of structures more massive than $\simeq 0.3 \mathrm{M}_{\mathrm{s}} \mathrm{pc}^{-1}$, appears to be smaller than the value expected from the power-law fitted above. This is most likely due to the finite size of the numerical experiment.

For $\log (M) \geq-3$, the total mass contained in a logarithmic interval of mass, follows approximately $\mathrm{d} M_{\mathrm{tot}} / \mathrm{d} \log M \propto$ $M^{2} \mathcal{N} \simeq M^{2-\beta} \simeq M^{0.25-0.3}$ which indicates that, although the mass of CNM is, in principle, dominated by the large clouds, the index of the power-law is nevertheless very shallow. This is confirmed by Fig. 16 which shows $\mathrm{d} M_{\text {tot }} / \mathrm{d} \log M$ as a function of $\log (M)$. It is seen that the mass in large structures is only slightly larger than the mass in hundred to thousand times smaller structures. This indicates that the description of the small structures is very important in order to achieve a fair description of the flow and its dynamics.

Complementary information is given in Fig. 17 which shows the total number of cells, $\mathrm{d} n_{\mathrm{c}, \text { tot }} / \mathrm{d} \log n_{\mathrm{c}}$, per logarithmic interval of computational cells number, $n_{\mathrm{c}}$, contained in the structure (expressed in cells of the highest resolution run). The physical size of the structures is obtained by taking the square root of the cell number and multiplying by $2 \times 10^{-3} \mathrm{pc}$ which is the numerical resolution of the $10000^{2}$ cells run. The size at which the structure number starts to decrease because of insufficient numerical resolution is about $10\left(\simeq \sqrt{n_{\mathrm{c}}}\right.$ for $\left.n_{\mathrm{c}} \simeq 100\right)$. The length 


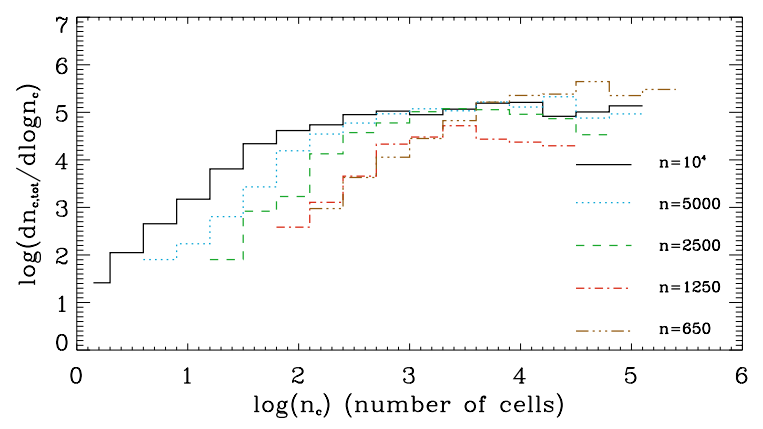

Fig. 17. Total number of cells per logarithmic interval of cells number (expressed in cells corresponding to the highest resolution run).

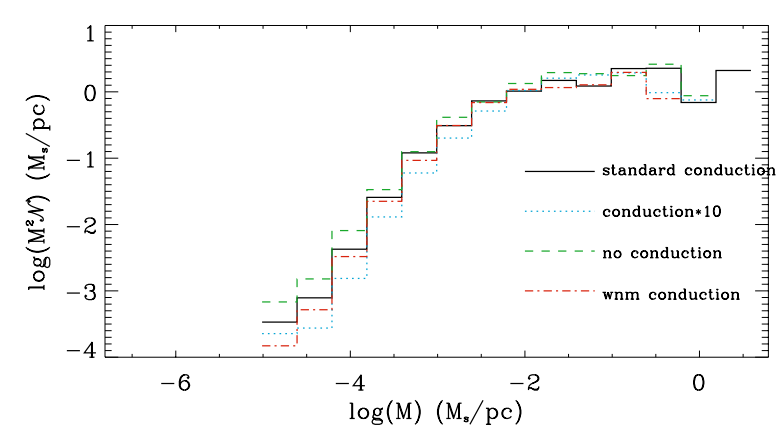

Fig. 18. Same as Fig. 16 except that the influence of various conductivity is shown.

of the bigger structures is roughly 300 cells $\left(\simeq \sqrt{n_{\mathrm{c}}}\right.$ for $\left.n_{\mathrm{c}} \simeq 10^{5}\right)$ which corresponds to about $0.6 \mathrm{pc}$.

\subsection{Influence of thermal conduction}

The importance of thermal conductivity has been recently pointed out by Koyama \& Inutsuka (2004). They show explicit cases for which the number of structures which forms do depend on the conductivity. In order to investigate the exact influence of the thermal conductivity on the structures, we have performed various runs in which its value has been changed. All runs have been performed with a resolution of $5000^{2}$ cells. The results are presented in Fig. 18. Four cases have been explored. The full black line is for standard ISM conduction, the dashed green line is when no explicit conduction is taken into account and the dotted blue line is for a conduction 10 times the standard ISM conditions. Finally, in order to determine the importance of the dependence in temperature of the conduction, we also perform a run in which the conduction does not vary with temperature and is equal to the conduction at $T=8000 \mathrm{~K}$. The corresponding run is shown by the dot-dashed red line. The number of structures more massive than $\simeq 10^{-3}$ solar mass per parsec appears to be independent of thermal conduction. There is a clear influence of thermal conduction on smaller structures, larger conduction runs produce less small structures than smaller conduction runs. This is qualitatively in good agreement with the trend expected from Field's linear analysis that shows that the conductivity prevents the formation of structures smaller than the Field length. However, the discrepancy between the different curves is not very large. We therefore conclude that thermal conduction, although not insignificant, does not appear to play a dominant rôle.

\section{Theoretical derivation of the mass spectrum}

Cloud mass spectrum is an important feature of the flow. It would be useful to have a theoretical model to explain the numerical results. Here, we propose an approach inspired from the Press \& Schecter (1974) formalism (see also Padmanabhan 1993).

\subsection{Principle and justification of the analysis}

The starting point of the Press \& Schecter (1974) formalism is to consider the density spectrum of the initial fluctuation that eventually lead to galaxies. They consider the density field smoothed at a given scale, $R$, by a window function $W$ and assume that the probability of finding a contrast density $\delta=\rho / \bar{\rho}-1, \bar{\rho}$ being the mean density, is

$P_{R}(\delta)=\frac{1}{\sqrt{2 \pi} \sigma(R)} \exp \left(\frac{-\delta^{2}}{2 \sigma(R)^{2}}\right)$

where

$\sigma^{2}(R)=\int \widetilde{\delta}^{2}(k) W_{k}^{2}(R) \mathrm{d}^{3} k$

(see e.g. Padmanabhan 1993, Chap. 5). In this last expression $\widetilde{\delta}^{2}(k)$ is the power-spectrum of $\delta$.

Therefore, the first question that must be addressed is whether this assumption is justified in the case of the atomic hydrogen. More precisely, since the CNM structures, are the final product of density fluctuations arising in the WNM, the question which has to be addressed is whether the spectrum of the density fluctuations in the WNM, smoothed at a scale $R$, is reasonably described by the probability stated by Eq. (5) and what is the value of $\sigma(R)$. One difficulty to address this question is that in the multiphase simulations presented previously, the WNM and the CNM are mixed together. They are therefore not representative of the initial state of the WNM which leads to the CNM structures. On the other hand, the early times of the simulation reflect the numerical setup rather than the initial conditions. To overcome this difficulty, we consider the simulation with cooling presented above but in which we have removed the CNM by taking $\min \left(\rho, \rho_{0}\right)$, with $\rho_{0}=3 \mathrm{~cm}^{-3}$ (see Sect. 5.4). As shown by Fig. 13, the power-spectrum of $\sqrt{\rho}$ is nearly Kolmogorov. We have checked that the power-spectrum of the density field is indeed nearly Kolmogorov as well. Another point is that the power-spectrum of the density in the isothermal simulation performed in Sect. 5.2 (let us remember that this simulation has exactly the same initial, boundary and forcing conditions than the other runs performed with cooling) is indeed very close to the Kolmogorov spectrum. The reason is that the isothermal simulation is subsonic (as for the simulation with cooling, the rms velocity is about half the sound speed of the WNM). Since in the ISM, the WNM is likely to be more or less isothermal until the thermal pressure is triggered to a value for which WNM is thermally unstable, this should constitute a reasonable description of WNM before the thermal transition occurs. Therefore, we assume that $\widetilde{\delta}^{2}(k) \propto k^{-n}$, with $n \simeq 8 / 3$.

To calculate the width of the distribution of the density fluctuations for the case of the turbulent WNM, we choose the simple window function sharply truncated in $k$-space, $W_{k}(R)=$ $\theta\left(R^{-1}-k\right)$ where $\theta(z)=1$ if $z \geq 0$ and 0 otherwise. With Eq. (6), it is easy to see that the integral diverges for small $k$. This is a natural consequence of the well known property of the Kolmogorov spectrum that most of the energy is on the large scales.We therefore have to introduce a lower value in $k$-space, 


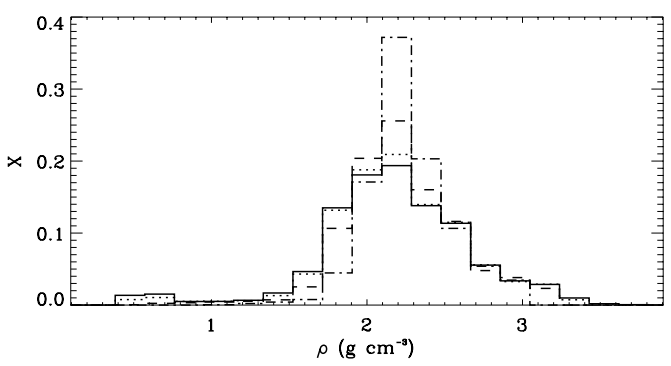

Fig. 19. Isothermal simulation. Probability distribution of the density field smoothed at scale $R=b \times \mathrm{d} x$, for various values of $b$. Solid line is $b=100$, dotted line is $b=200$, dashed line is $b=500$, whereas dot-dashed line is $b=1000$.

which corresponds to an upper limit of the spatial scales, $L_{\mathrm{c}}$. In the case of the simulation, this is naturally given by the size of the computational box whereas in the ISM, this would be the energy injection scale. We have:

$\sigma^{2}(R)=\int_{1 / L_{\mathrm{c}}}^{1 / R} C k k^{-n} \mathrm{~d} k=\frac{C}{n-2}\left(\left(\frac{1}{L_{\mathrm{c}}}\right)^{2-n}-\left(\frac{1}{R}\right)^{2-n}\right)$,

where $C$ is a constant.

The question is then, are the density fluctuations reasonably described by Eqs. (5) and (7)? To answer this question, we have smoothed the density field of the isothermal and cooling simulations using the simple window function, $W_{b}\left(\rho_{i, j}\right)=$ $1 / b^{2} \sum_{|i-m| \leq b,|j-n| \leq b} \rho_{m, n}$, for various values of $b$. Figures 19 and 20 show the results in the isothermal case and in the case with cooling (since the isothermal simulation has $5000^{2}$ cells, the values of $b$ are 2 times lower than in the case with cooling). It is seen that, in the isothermal case, the probability distribution is reasonably Gaussian. As expected from Eq. (7), $\sigma$ appears to be a decreasing function of $b=R / \mathrm{d} x$. In the case with cooling, the situation is slightly less clear because the shape is a little skewed towards the large densities. This is a consequence of the fact that a fraction of the gas is thermally unstable and in transition towards CNM or is already CNM. This dense gas is therefore on the process of condensation or already condensed and should not be considered in the pdf of density fluctuations of WNM.

Beyond this qualitative agreement, we present in Appendix A a more quantitative estimate that confirms the validity of Eq. (7). Thus it appears that the assumption of the distribution of the density fluctuations being given by Eqs. (5) and (7) is sufficiently justified.

\subsection{Mass function}

To calculate the mass function, we can now follow the same procedure as for the cosmological calculation. We note, however, two important differences. First, since we assume statistical stationarity, there is no time dependence in our analysis. Second, the collapse is not due to gravity but to thermal instability.

Here, since our primary goal is to understand the simulation results, we consider the $2 \mathrm{D}$ case. Thus, for a perturbation of size, $L$, the final mass of the structures is about $M(L)=\bar{\rho} L^{2}$, Eq. (7) is equivalent to

$\sigma^{2}(M)=\frac{C}{n-2}\left(M\left(L_{\mathrm{c}}\right)^{(n-2) / 2}-M^{(n-2) / 2}\right) \bar{\rho}^{(2-n) / 2}$,

where $M=M(R)$.

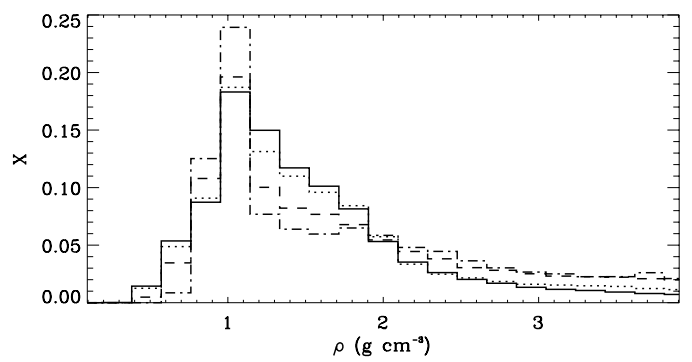

Fig. 20. 2-phase simulation. Probability distribution of the density field smoothed at scale $R=b \times \mathrm{d} x$, for various values of $b$. Solid line is $b=200$, dotted line is $b=500$, dashed line is $b=1000$, whereas dot-dashed line is $b=2000$.

The fraction of objects having a mass larger than $M$ is thus given by:

$F(M)=\int_{0}^{\infty} P_{R}(\delta) s(\delta) \mathrm{d} \delta$

where $s$ is the selection function ${ }^{2}$. The selection function, $s$, determines the fluctuations that will eventually lead to the formation of a CNM structure. For simplicity, we will assume that once the density reaches a threshold $\delta_{\mathrm{c}}$, it leads to a thermal collapse and to the formation of a CNM structure. Although this assumption is reasonable given the physical characteristics of thermal instability, we note that various improvements could certainly be possible, as for example considering a density-pressure relation for the instability threshold or taking into account the stabilizing rôle of the turbulence within the perturbation (in that case $s$ would also depend on the velocity dispersion). Thus we have

$F(M)=\int_{\delta_{\mathrm{c}}}^{\infty} P_{R}(\delta) \mathrm{d} \delta$

The mass function, $\mathcal{N}(M)$, is then simply given by $-\partial F / \partial M /(M / \bar{\rho})$. We have ${ }^{3}$

$\mathcal{N}(M) \mathrm{d} M=-\frac{1}{\sqrt{2 \pi}} \frac{\bar{\rho}}{M} \frac{\delta_{\mathrm{c}}}{\sigma^{2}} \frac{\mathrm{d} \sigma}{\mathrm{d} M} \exp \left(\frac{-\delta_{\mathrm{c}}^{2}}{2 \sigma^{2}}\right) \mathrm{d} M$.

With Eq. (8), we get

$$
\begin{aligned}
\frac{\mathrm{d} \sigma}{\mathrm{d} M}= & -\sqrt{C} \bar{\rho}^{1 / 2-n / 4} \times \frac{M^{(n-2) / 2}}{4 M} \\
& \times \sqrt{\frac{(n-2)}{\left(M\left(L_{\mathrm{c}}\right)^{(n-2) / 2}-M^{(n-2) / 2}\right)}}
\end{aligned}
$$

This leads to:

$\mathcal{N}(M) \mathrm{d} M \propto M^{(n-2) / 2-2} \frac{1}{\sigma^{3}} \exp \left(-\delta_{\mathrm{c}}^{2} /\left(2 \sigma^{2}\right)\right) \mathrm{d} M$.

Therefore for small masses, i.e. mass smaller than $M\left(L_{\mathrm{c}}\right)$, we obtain that $\mathcal{N}(M) \propto M^{(n-2) / 2-2}$ with $n \simeq 8 / 3$, we get $\mathcal{N}(M) \propto$ $M^{-5 / 3}$. We note that $5 / 3 \simeq 1.666$ is rather close to the value 1.7

2 We note that in cosmology this expression is known to be inconsistent because in the cosmological case, the integral of $\mathrm{d} F=f(m) \mathrm{d} m$ from 0 to $\infty$ should be equal to 1 . This problem has been solved by the Excursion set theory (Bond et al. 1991). The results for the shape of the mass spectrum is however independent of this and we will not consider this problem further at this stage.

3 The Excursion set theory would give the same result multiplied by a factor 2 . 
obtained in the simulation. For a mass close to $M\left(L_{\mathrm{c}}\right)$, there is an exponential cutoff which is due to the cut, $L_{\mathrm{c}}$ introduced at large scales. Altogether these results are similar to the results obtained in the cosmological case. An important difference however lies in the exponent of the mass spectrum which is less stiff. This is a consequence of the fact that in a turbulent flow, most of the energy is at large scales implying more large scale fluctuations.

\subsection{The $3 D$ case: similarity with the CO clumps}

This analysis can be straightforwardly applied to the 3D case. The only differences are that $n=11 / 3$ (since the simulations presented here are bidimensional, it is worth to remember that Kim \& Ryu (2005) do find that in transonic isothermal simulations, the density power spectrum is nearly Kolmogorov) and $M=\bar{\rho} R^{3}$. We obtain:

$$
\frac{\mathrm{d} \sigma}{\mathrm{d} M} \propto-\frac{M^{(n-3) / 3}}{M} \sqrt{\frac{(n-3)}{\left(M\left(L_{\mathrm{c}}\right)^{(n-3) / 3}-M^{(n-3) / 3}\right)}},
$$

and

$\mathcal{N}(M) \mathrm{d} M \propto M^{(n-3) / 3-2} \frac{1}{\sigma^{3}} \exp \left(-\delta_{\mathrm{c}}^{2} /\left(2 \sigma^{2}\right)\right) \mathrm{d} M$.

The index of the power law of the mass spectrum in the $3 \mathrm{D}$ case and for masses small compared to $M\left(L_{\mathrm{c}}\right)$, is about $2-(n-3) / 3=$ $16 / 9 \simeq 1.78$, i.e. slightly stiffer than the value inferred in the $2 \mathrm{D}$ case.

Interestingly, we note that the value inferred here, turns out to be similar to the index of the mass spectrum observed for the CO clumps of masses between $10^{4}$ solar mass and one Jupiter mass (Kramer et al. 1998; Heithausen et al. 1998).

Although it cannot be excluded that this is a pure coincidence, one possibility is that the origin of the $\mathrm{CO}$ clumps is indeed rooted in the atomic gas. The mass distribution of the $\mathrm{CO}$ clumps may therefore be determined during the atomic phase before the gas becomes molecular.

\section{Conclusion}

We have presented high resolution numerical simulations aiming to describe a turbulent interstellar atomic flow. The high resolution $\left(2 \times 10^{-3} \mathrm{pc}\right)$ provides a good description (although not sufficient to reach numerical convergence) of the flow close to the spatial scales of the smallest structures observed in HI. We confirm the main results obtained in Paper I. The flow is very fragmented and the phases are tightly interwoven. The CNM structures are connected to the WNM by stiff thermal fronts and are locally in near pressure equilibrium with the surrounding WNM. Altogether, this is reminiscent of the classical 2-phase model in spite of the fact that the flow is dynamical and turbulent.

We find that small scale structures, either diffuse or very dense, are naturally produced in a turbulent 2-phase medium. Whereas the former are simply the tail of the structure mass spectrum induced by turbulence, the latter, which are somehow reminiscent of the TSAS observed in the atomic gas, are transient events produced by supersonic collisions between CNM fragments.

We characterize the flow by studying various statistical quantities, namely the mass distribution of the CNM structures, the velocity and density power-spectrum and the energy spectrum, paying special attention to the numerical resolution. We stress that the present problem requires a lot of numerical resolutions to obtain reliable results. Typically at least $5000^{2}$ to $10000^{2}$ cells are needed and even so, no strict convergence is achieved.

Our main results are as follows.

For structures of size larger than about 10 grid cells, for which numerical diffusivity is not too important, we find a mass spectrum, $\mathcal{N}(M) \propto M^{-1.7}$. In order to explain this result, we have carried out a calculation based on Press \& Schecter (1974) formalism. Our theory predicts $\mathcal{N}(M) \propto M^{-5 / 3}$ in 2D and $\mathcal{N}(M) \propto M^{-16 / 9}$ in $3 \mathrm{D}$. One of the main assumptions is that the CNM clumps are due to density fluctuations within WNM, which behaves as a sub to transonic nearly isothermal gas. We note that these mass spectra are similar to the mass spectrum inferred observationally for CO clumps. This is compatible with the origin of CO clumps being rooted in the very diffuse atomic gas although this does not, by any means, constitute a proof.

Like in highly supersonic isothermal flows, the density power-spectrum is rather flat. However, the structures being pressure-bounded and not bounded by shocks as in supersonic isothermal simulations, they are long-living objects. Unlike supersonic isothermal simulations, the velocity power-spectrum follows the Kolmogorov prediction and is dominated by its solenoidal part. At scales larger than about $1 \mathrm{pc}$, most of the energy is in the WNM whereas at scales smaller than about $1 \mathrm{pc}$, it is mostly in the CNM. Indeed, the energy is nearly equally distributed in $k$-space for scales ranging between about 2 and $2 \times$ $10^{-2} \mathrm{pc}$ where numerical diffusion becomes dominant. This behaviour is due to the density structure of the flow that presents relatively flat density power-spectrum, indicating that in a thermally bistable flow like the one we studied, the kinetic energy at scale below $1 \mathrm{pc}$, appears to be dominated by the translational motions of the CNM fragments.

We also study the influence of the thermal conduction by performing various runs with different thermal conductivities and find a modest influence, mainly on the small structures.

In a companion paper (Paper III), we further characterize the simulation results by studying line of sight statistics and the physical properties of the CNM clouds. We also calculate synthetic HI spectra and reach the conclusion that, although the lines of sight from which they are calculated are very complex, showing in some cases several structures, the spectra are relatively smooth and simply broadened by the complex motions along the line of sight.

Altogether these results suggest that the turbulence which takes place in the neutral atomic interstellar gas is very different from the turbulence which takes place in an isothermal or polytropic gas. One important question that remains to be investigated is how these conclusions will change in a more realistic framework of 3D flows. To answer this question 3D simulations have been performed and will be presented elsewhere (Audit \& Hennebelle 2007).

Acknowledgements. We acknowledge the support of the CEA computing center, CCRT, where all the simulations where carried out. P.H. is most grateful to John Scalo for stimulating exchanges on the interstellar turbulence as well as insightful comments. We thank Snezana Stanimirović, the referee, for constructive and interesting comments which have improved the paper significantly.

\section{Appendix A: Further measurement of the pdf of density fluctuations}

In order to measure more accurately the dependence of $\sigma$ (Eq. (7)) on $R$, we define $\delta \rho_{b}-\delta \rho_{2 b}=W_{b}(\rho)-$ $W_{2 b}(\rho)$, i.e. the density field smoothed by the window function $W_{b}-W_{2 b}$. With this window function, one finds that 

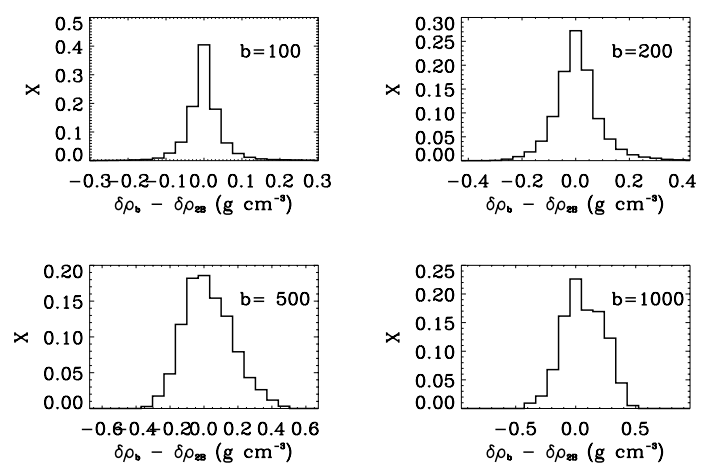

Fig. A.1. Isothermal simulation. Probability distribution of the density field smoothed by the window function $W_{b}-W_{2 b}$ for various value of $b$. The range of the $x$-axis being proportional to $b^{n-2}$, it appears that the length of these distribution is reasonably Gaussian and roughly proportional to $b^{n-2}$.
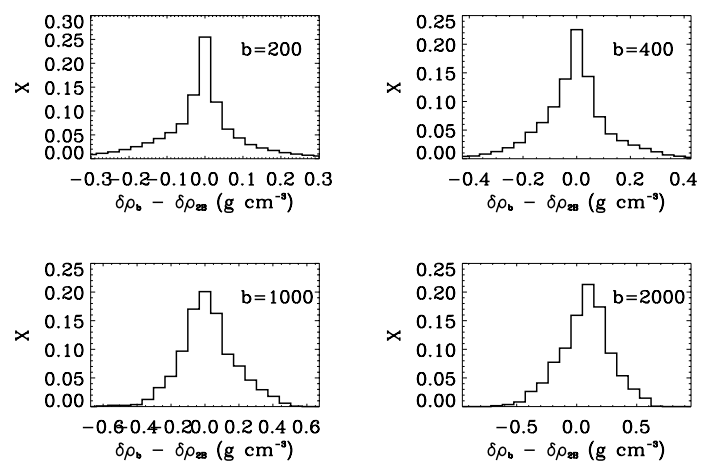

Fig. A.2. Same as Fig. A.1 for the 2-phase simulation in which the density field has been truncated by taking $\min \left(\rho, \rho_{0}\right)$.

$\sigma(R)=C /(n-2) \times\left(2^{n-2}-1\right) R^{n-2}$. In particular, it is independent of $L_{\mathrm{c}}$ and is proportional to $R^{n-2}=R^{2 / 3}$ for $n=8 / 3$.

Figures A.1 and A.2 display the distribution of $\delta \rho_{b}-\delta \rho_{2 b}$ for various values of $b$ for the isothermal and the 2-phase simulations. The range of the $x$-axis is proportional to $b^{n-2}$. As can be seen, the distributions are reasonably Gaussian and the width is broadly proportional to $b^{n-2}$ for both cases except for small $b$ in the isothermal simulation.

\section{References}

Audit, E., \& Hennebelle, P. 2005, A\&A, 433, 1 (Paper I) Audit, E., \& Hennebelle, P. 2007, in preparation

Beresnyak, A., Lazarian, A., \& Cho, J. 2005, ApJ, 624, L93

Bond, J., Cole, S., Efstathiou, G., \& Kaiser, N. 1991, ApJ, 379, 440

Braun, R., \& Kanekar, N. 2005, A\&A, 436, L53
Crovisier, J. 1981, A\&A, 94, 162

de Avillez, M., \& Breitschwerdt, D. 2005, A\&A, 436, 585

Dib, S., \& Burkert, A. 2005, A\&A, 630, 238

Dickey, J., \& Lockman, F. 1990, ARA\&A, 28, 215

Field, G. 1965, ApJ, 142, 531

Field, G., Goldsmith, D., \& Habing, H. 1969, ApJ, 155, L149

Folini, D., \& Walder, R. 2006, A\&A, 459, 1

Gazol, A., Vázquez-Semadeni, E., Sánchez-Salcedo, F., \& Scalo, J. 2001, ApJ, 557, L124

Gazol, A., Vázquez-Semadeni, E., \& Kim, J. 2005, ApJ, 630, 911

Heiles, C. 1997, ApJ, 481, 193

Heiles, C., \& Troland, T. 2003, ApJ, 586, 1067

Heiles, C., \& Troland, T. 2005, ApJ, 624, 773

Heithausen, A., Bensch, F., Stutzki, J., Falgarone, F., \& Panis, J.-F. 1998, A\&A, 331, L65

Heitsch, F., Burkert, A., Hartmann, L., Slyz, A., \& Devriendt, J. 2005, ApJ, 633, 113

Heitsch, F., Slyz, A., Devriendt, J., Hartmann, L., \& Burkert, A. 2006, ApJ, 648, 1052

Hennebelle, P., \& Pérault, M. 1999, A\&A, 351, 309

Hennebelle, P., \& Pérault, M. 2000, A\&A, 359, 1124

Hennebelle, P., \& Passot, T. 2006, A\&A, 448, 1083

Hennebelle, P., Audit, E., \& Miville-Deschènes, M.-A. 2007, A\&A, 465, 445 (Paper III)

Kim, J., \& Ryu, D. 2005, ApJ, 630, L45

Klessen, R., Heitsch, F., \& Mac Low, M.-M. 2000, ApJ, 535, 887

Kramer, C., Stutzki, J., Rohrig, R., \& Corneliussen, U. 1998, A\&A, 329, 249

Kolmogorov, A. N. 1941, Proc. R. Soc. London Ser. A, Reprinted in 1991, 434

Koyama, H., \& Inutsuka, S. 2000, ApJ, 532, 980

Koyama, H., \& Inutsuka, S. 2002, ApJ, 564, L97

Koyama, H., \& Inutsuka, S. 2004, ApJ, 602, L25

Koyama, H., \& Inutsuka, S. 2006, ApJ, in press [arXiv: astro-ph/0605528]

Kraichnan, R. H. 1967, Phys. Fluid, 8, 1385

Kritsuk, A. G., \& Norman, M. L. 2002, ApJ, 569, L127

Kulkarni, S. R., \& Heiles, C. 1987, Interstellar processes, ed. D. Hollenbach, \& H. Thronson (Reidel)

Miville-Deschênes, M.-A., Joncas, G., Falgarone, E., \& Boulanger, F. 2003, A\&A, 411, 109

Padmanabhan, T. 1993, Structure formation in the universe (Cambridge university press)

Padoan, P., Jimenez, R., Juvela, M., \& Nordlund, A. 2004, ApJ, 604, 49

Passot, T., Pouquet, A., \& Woodward, P. 1988, A\&A, 197, 228

Passot, T., \& Vazquez-Semadeni, E. 1998, Phys. Rev. E., 58, 4501

Penston, M., \& Brown, F. 1970, MNRAS, 150, 373

Piontek, R., \& Ostriker, E. 2004, ApJ, 601, 905

Press, W., \& Schecter, P. 1974, ApJ, 187, 425

Sánchez-Salcedo, F. J., Vázquez-Semadeni, E., \& Gazol, A. 2002, ApJ, 577, 768

Scalo, J., Vazquez-Semadeni, E., Chappell, D., \& Passot, T. 1998, 504, 835

Stanimirović, S., \& Heiles, C. 2005, ApJ, 631, 371

Toro, E. 1997, Riemann solvers and numerical methods for fluid dynamics (Springer)

Vázquez-Semadeni, E., Ryu, D., Passot, T., González, R., \& Gazol, A. 2006, ApJ, 643, 245

Vishniac, E. 1994, ApJ, 428, 186

Walder, R., \& Folini, D. 1996, A\&A, 315, 265

Walder, R., \& Folini, D. 1998, A\&A, 330, L23

Wolfire, M. G., Hollenbach, D., \& McKee, C. F. 1995, ApJ, 443, 152

Wolfire, M. G., Hollenbach, D., \& McKee, C. F. 2003, ApJ, 587, 278

Zel'dovich, Y., \& Pikel'ner, S. 1969, JETP, 29, 170 